\title{
Expand, Enlarge, and Check New algorithms for the coverability problem of WSTS ${ }^{\star}$
}

\author{
Gilles Geeraerts, Jean-François Raskin, and Laurent Van Begin^^ \\ DI, Université Libre de Bruxelles
}

\begin{abstract}
In this paper, we present a general algorithmic schema called "Expand, Enlarge and Check" from which new efficient algorithms for the coverability problem of WSTS can be constructed. We show here that our schema allows us to define forward algorithms that decide the coverability problem for several classes of systems for which the Karp and Miller procedure cannot be generalized, and for which no complete forward algorithms were known. Our results have important applications for the verification of parameterized systems and communication protocols.
\end{abstract}

\section{Introduction}

Model-checking is nowadays widely accepted as a powerful technique for the automatic verification of reactive systems that have natural finite state abstractions. However, many reactive systems are only naturally modelled as infinitestate systems. Consequently, a large (and successful) research effort has recently focused on the application of model-checking techniques to infinite-state models such as FIFO channel systems [2], Petri nets [15], broadcast protocols [7], etc.

One of the positive results is the decidability of the coverability problem for well-structured transition systems (WSTS for short). WSTS enjoy an infinite set of states that is well-quasi ordered by $\leq$ and their transition relation is monotonic w.r.t $\leq$. Examples of such systems are Petri nets and their monotonic extensions $[5,15]$, broadcast protocols [8], lossy channel systems [2]. The coverability problem asks, given two states $c_{1}$ and $c_{2}$, whether there is $c_{3} \geq c_{2}\left(c_{3}\right.$ covers $\left.c_{2}\right)$ that is reachable from $c_{1}$.

A general algorithm (i.e. a procedure that always terminates) is known to solve the coverability problem for WSTS $[1,10]$. It symbolically manipulates upward-closed sets of states, obtained by unrolling the transition relation in a backward fashion. Unfortunately, backward search is seldom efficient in practise [12], and the only complete forward approach known so far is the Karp-Miller algorithm that can only be applied to a small subclass of WSTS: Petri nets.

The Karp and Miller procedure computes, through a combination of a forward exploration strategy and a simple acceleration technique, the so-called covering set of the net, which is known to be well-suited to decide the coverability problem. After several attempts to generalize this procedure to WSTS

\footnotetext{
* This research has been partially supported by the FRFC grant 2.4530.02.

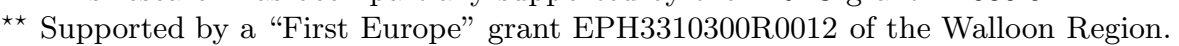


(which have all produced incomplete approaches $[8,9]$ ), it has been shown in $[6]$ that Petri nets form the sole class (among the examples cited above) for which the covering set is constructible in general. However, this set always exists and is usually finitely representable. Our main contribution is to make the best of this fact and devise a forward technique that is complete to decide the coverability problem for a large class of WSTS. This class includes, among others, all the monotonic extensions of Petri nets defined in the literature, as well as lossy channel systems.

We present a new schema of algorithm: "Expand, Enlarge and Check" that works by iteratively constructing more and more precise abstractions of the system. These abstractions (made up of reachable states and limit elements) are guaranteed to become precise enough to decide the coverability problem after a finite number of steps. We show how to apply the schema on two classes of WSTS of practical interest: monotonic extensions of Petri nets (that are useful to model parameterized systems $[11,15]$ ) and lossy channels systems (that are useful to model communication protocols [2]).

Due to lack of space, most of the proofs have been omitted. A complete version of the paper can be found at:

http://www.ulb.ac.be/di/ssd/cfv/TechReps/TechRep_CFV_2004_25.pdf

\section{Preliminaries}

In this section, we recall some fundamental results about well-quasi orderings and well-structured transition systems (the systems we analyze here). We show how to finitely represent upward- and downward-closed sets of states (which will allow us to devise symbolic algorithms), and discuss And-Or graphs (useful to represent abstractions of systems).

Well quasi-orderings and adequate domains of limits A well quasi ordering $\leq$ on the elements of a set $C$ (wqo for short) is a reflexive and transitive relation such that for any infinite sequence $c_{0} c_{1} \ldots c_{n} \ldots$ of elements in $C$, there exist two indices $i$ and $j$, such that $i<j$ and $c_{i} \leq c_{j}$. In the following, we note $c_{i}<c_{j}$ if $c_{i} \leq c_{j}$ but $c_{j} \not \leq c_{i}$.

Let $\langle C, \leq\rangle$ be a well-quasi ordered set. A $\leq$-upward closed set $U \subseteq C$ is such that for any $c \in U$, for any $c^{\prime} \in C$ such that $c \leq c^{\prime}, c^{\prime} \in U$. A $\leq$ downward closed set $D \subseteq C$ is such that for any $c \in D$, for any $c^{\prime} \in C$ such that $c^{\prime} \leq c, c^{\prime} \in D$. It is well-known that any $\leq$-upward closed set $U \subseteq C$ is uniquely determined by its finite sets of minimal elements. Formally, the set of $\leq-$ minimal elements $\operatorname{Min}(U)$ of a set $U \subseteq C$ is a minimal set such that $\operatorname{Min}(U) \subseteq U$ and $\forall s^{\prime} \in U: \exists s \in \operatorname{Min}(U): s \leq s^{\prime}$. The next proposition is a consequence of wqo:

Proposition 1. Let $\langle C, \leq\rangle$ be a wqo set and $U \subseteq C$ be an $\leq$-upward closed set, then: $\operatorname{Min}(U)$ is finite and $U=\left\{c \mid \exists c^{\prime} \in \operatorname{Min}(U): c^{\prime} \leq c\right\}$.

Thus, any $\leq$-upward closed set can be effectively represented by its finite set of minimal elements. To obtain a finite representation of downward-closed sets, 
we must use well-chosen limit elements $\ell \notin C$ to represent downward closures of infinite increasing chains of elements. Thus, we introduce the notion of adequate domain of limits.

Definition 1. Let $\langle C, \leq\rangle$ be a well-quasi ordered set and $L$ be a set of elements disjoint from $C$, the tuple $\langle L, \sqsubseteq, \gamma\rangle$ is called an adequate domain of limits for $\langle C, \leq\rangle$ if the following conditions are satisfied: $\left(\mathrm{L}_{1}\right.$ : representation mapping $) \gamma$ : $L \cup C \rightarrow 2^{C}$ associates to each element in $L \cup C$ a $\leq$-downward closed set $D \subseteq C$, furthermore, for any $c \in C$, we impose that $\gamma(c)=\left\{c^{\prime} \mid c^{\prime} \leq c\right\}$. In the following, $\gamma$ is extended to sets $\mathcal{S} \subseteq L \cup C$ in the natural way: $\gamma(\mathcal{S})=\cup_{c \in \mathcal{S}} \gamma(c) ;\left(\mathrm{L}_{2}\right.$ : top element) There exists a special element $T \in L$ such that $\gamma(T)=C ;\left(\mathrm{L}_{3}\right.$ : precision order) The elements of $C \cup L$ are ordered by the complete quasi order $\sqsubseteq$, defined as follows: $d_{1} \sqsubseteq d_{2}$ if and only if $\gamma\left(d_{1}\right) \subseteq \gamma\left(d_{2}\right)$; ( $\mathrm{L}_{4}$ : completeness) for any downward closed set $D \subseteq C$, there exists a finite set $D^{\prime} \subseteq C \cup L$ with $\gamma\left(D^{\prime}\right)=D$.

Well-structured transition systems and coverability problem A transition system is a tuple $S=\left\langle C, c_{0}, \rightarrow\right\rangle$ where $C$ is a (possibly infinite) set of states, $c_{0} \in C$ is the initial state, $\rightarrow \subseteq C \times C$ is a transition relation. In the following, $c \rightarrow c^{\prime}$ will denote that $\left\langle c, c^{\prime}\right\rangle \in \rightarrow$. For any state $c$, Post $(c)$ denotes the set of onestep successors of $c$, i.e. Post $(c)=\left\{c^{\prime} \mid c \rightarrow c^{\prime}\right\}$. We require $\operatorname{Post}(c) \neq \emptyset$ for any $c \in C^{1}$. This operator is extended to sets of states $C^{\prime} \subseteq C$ as follows: $\operatorname{Post}\left(C^{\prime}\right)=\left\{c \mid \exists c^{\prime} \in C^{\prime}: c^{\prime} \rightarrow c\right\}$. A path of $S$ is a sequence of states $c_{1}, c_{2}, \ldots, c_{k}$ such that $c_{1} \rightarrow c_{2} \rightarrow \cdots \rightarrow c_{k}$. A state $c^{\prime}$ is reachable from a state $c$, noted $c \rightarrow^{*} c^{\prime}$, if we have a path $c_{1}, c_{2}, \ldots c_{k}$ in $S$ with $c_{1}=c$ and $c_{k}=c^{\prime}$. Given a transition system $S=\left\langle C, c_{0}, \rightarrow\right\rangle$, Reach $(S)$ denotes the set $\left\{c \in C \mid c_{0} \rightarrow^{*} c\right\}$.

Definition 2. A transition system $S=\left\langle C, c_{0}, \rightarrow\right\rangle$ is a well-structured transition system for the quasi order $\leq \subseteq C \times C$ if the two following properties hold: $\left(\mathrm{W}_{1}\right.$ : well-ordering $) \leq$ is a well-quasi ordering and $\left(\mathrm{W}_{2}\right.$ : monotonicity) for all $c_{1}, c_{2}, c_{3} \in C$ such that $c_{1} \leq c_{2}$ and $c_{1} \rightarrow c_{3}$, there exists $c_{4} \in C$ such that $c_{3} \leq c_{4}$ and $c_{2} \rightarrow c_{4}$.

From now on, $S=\left\langle C, c_{0}, \rightarrow, \leq\right\rangle$ will denote the well-structured transition system $\left\langle C, c_{0}, \rightarrow\right\rangle$ for $\leq$. In the sequel, we need to manipulate WSTS and adequate domain of limits. In particular, we need the following effectiveness properties:

Definition 3. A WSTS $S=\left\langle C, c_{0}, \rightarrow, \leq\right\rangle$ and an adequate domain of limits $\langle L, \sqsubseteq, \gamma\rangle$ are effective if the following conditions are satisfied: $\left(\mathrm{E}_{1}\right) C$ and $L$ are recursively enumerable; $\left(\mathrm{E}_{2}\right)$ for any $c_{1}, c_{2} \in C$, we can decide whether $c_{1} \rightarrow c_{2}$; $\left(\mathrm{E}_{3}\right)$ for any two finite subsets $C^{\prime} \subseteq C$ and $L^{\prime} \subseteq L$, for any $d \in C^{\prime} \cup L^{\prime}$ and any finite subset $D \subseteq C^{\prime} \cup L^{\prime}$, we can decide whether Post $(\gamma(d)) \subseteq \gamma(D)$; $\left(\mathrm{E}_{4}\right)$ For any finite subsets $D_{1}, D_{2} \subseteq C \cup L$, we can decide whether $\gamma\left(D_{1}\right) \subseteq \gamma\left(D_{2}\right)$.

Problem 1. The coverability problem for well-structured transition systems is defined as follows: "Given a well-structured transition system $S$ and the $\leq$-upward closed set $U \subseteq C$, determine whether $\operatorname{Reach}(S) \cap U \neq \emptyset$ ?"

\footnotetext{
${ }^{1}$ Note that this condition is not restrictive since we can always add a transition to a dummy state.
} 
To solve the coverability problem, we use covering sets, defined as follows:

Definition 4. Let $S=\left\langle C, c_{0}, \rightarrow, \leq\right\rangle$ be a WSTS. The covering set of $S$, noted Cover $(S)$, is the (unique) smallest subset of $C$ which $\left(\mathrm{CS}_{1}\right)$ is $\leq$-downward closed and $\left(\mathrm{CS}_{2}\right)$ contains $\operatorname{Reach}(S)$.

Property For any WSTS $S=\left\langle C, c_{0}, \rightarrow, \leq\right\rangle$ with an adequate domain of limits $\langle L, \sqsubseteq, \gamma\rangle$ for $\langle C, \leq\rangle$, by property $\mathrm{L}_{4}$ of Definition 1 , there exists a finite subset $\mathrm{CS}(S) \subseteq L \cup C$ such that $\gamma(\mathrm{CS}(S))=\operatorname{Cover}(S)$. In the following, $\operatorname{CS}(S)$ is called a coverability set of the covering set $\operatorname{Cover}(S)$ and finitely represents that set.

Proposition 2. For any WSTS $S=\left\langle C, c_{0}, \rightarrow, \leq\right\rangle$, the covering set of $S$ is such that for any $\leq$-upward closed set $U \subseteq C: \operatorname{Reach}(S) \cap U=\emptyset$ iff $\operatorname{Cover}(S) \cap U=\emptyset$.

And-Or graph and its avoidability problem An And-Or graph is a tuple $G=$ $\left\langle V_{A}, V_{O}, v_{i}, \Rightarrow\right\rangle$ where $V=V_{A} \cup V_{O}$ is the set of nodes ( $V_{A}$ is the set of "And" nodes and $V_{O}$ is the set of "Or" nodes), $V_{A} \cap V_{O}=\emptyset, v_{i} \in V_{O}$ is the initial node, and $\Rightarrow \subseteq\left(V_{A} \times V_{O}\right) \cup\left(V_{O} \times V_{A}\right)$ is the transition relation such that for any $v \in V_{A} \cup V_{O}$, there exists $v^{\prime} \in V_{A} \cup V_{O}$ such that $\left(v, v^{\prime}\right) \in \Rightarrow$.

Definition 5. A compatible unfolding of an And-Or graph $G=\left\langle V_{A}, V_{O}, v_{i}, \Rightarrow\right\rangle$ is an infinite labelled tree $T_{G}=\langle N$, root, $B, \Lambda\rangle$ where: $(i) N$ is the set of nodes of $T_{G},($ ii $)$ root $\in N$ is the root of $T_{G}$, (iii) $B \subseteq N \times N$ is the transition relation of $T_{G}$, (iv) $\Lambda: N \rightarrow V_{A} \cup V_{0}$ is the labelling function of the nodes of $T_{G}$ by nodes of $G$ that respects the three following compatibility conditions ( $\Lambda$ is extended to sets of nodes in the usual way): $\left(\mathrm{C}_{1}\right) \Lambda$ (root) $=v_{i} ;\left(\mathrm{C}_{2}\right)$ for all $n \in N$ such that $\Lambda(n) \in V_{A}$, we have that $(a)$ for all nodes $v^{\prime} \in V_{O}$ such that $\Lambda(n) \Rightarrow v^{\prime}$, there exists one and only one $n^{\prime} \in N$ such that $B\left(n, n^{\prime}\right)$ and $\Lambda\left(n^{\prime}\right)=v^{\prime}$, and conversely (b) for all nodes $n^{\prime} \in N$ such that $B\left(n, n^{\prime}\right)$, there exists $v^{\prime} \in V_{O}$ such that $\Lambda(n) \Rightarrow v^{\prime}$ and $\Lambda\left(n^{\prime}\right)=v^{\prime} .\left(\mathrm{C}_{3}\right)$ for all $n \in N$ such that $\Lambda(n) \in V_{O}$, we have that: there exists one and only one $n^{\prime} \in N$ such that $B\left(n, n^{\prime}\right)$, and $\Lambda(n) \Rightarrow \Lambda\left(n^{\prime}\right)$.

Problem 2. The And-Or Graph Avoidability Problem is defined as follows: "Given an And-Or graph $G=\left\langle V_{A}, V_{O}, v_{i}, \Rightarrow\right\rangle$ and a set $E \subseteq V_{A} \cup V_{O}$, does there exist $T=\langle N$, root $, \Lambda, B\rangle$, a compatible unfolding of $G$, such that $\Lambda(N) \cap E=\emptyset$ ?". When the answer is positive, we say that $E$ is avoidable in $G$.

It is well-known that this problem is complete for PTIME.

\section{A new schema of algorithms}

In this section, we introduce our new schema of algorithms to decide the coverability problem for WSTS. We first explain, in subsection 3.1, how to build an abstraction of a given WSTS, w.r.t. a given finite set of reachable states $C^{\prime} \subseteq C$ and a given finite set of limit elements $L^{\prime} \subseteq L$. These abstractions are And-Or graphs whose nodes are annotated by downward-closed sets of states of a WSTS. We show in subsection 3.2 that any unfolding of this And-Or graph is able to 
simulate the behaviours of its associated WSTS (Proposition 3). Moreover, if the downward-closed sets that are used to annotate the And-Or graph are precise enough (in a sense that we make clear in Theorem 2), then the And-Or graph can be used to decide negative instances of the coverability problem. Based on those results, we propose a new algorithmic schema to decide the coverability problem of WSTS. It works by iteratively constructing abstractions of the WSTS which become more and more precise. In parallel, it also explores, in a breadthfirst fashion, the set of reachable states of the system (to be able to decide the positive instances of the problem). Thus, after a finite number of steps either a concrete trace to a covering state will be found, or precise enough abstraction will be computed to prove that no covering state can ever be reached.

\subsection{The And-Or Graph $\operatorname{Abs}\left(S, C^{\prime}, L^{\prime}\right)$}

Definition 6. Given a WSTS $S=\left\langle C, c_{0}, \rightarrow, \leq\right\rangle$, an adequate domain of limits $\langle L, \sqsubseteq, \gamma\rangle$ for $\langle C, \leq\rangle$, a finite subset $C^{\prime} \subseteq C$ with $c_{0} \in C^{\prime}$, and a finite subset $L^{\prime} \subseteq L$ with $\top \in L^{\prime}$, the And-Or graph $G=\left\langle V_{A}, V_{O}, v_{i}, \Rightarrow\right\rangle$, noted $\operatorname{Abs}\left(S, C^{\prime}, L^{\prime}\right)$, is defined as follows: $\left(\mathrm{A}_{1}\right) V_{O}=C^{\prime} \cup L^{\prime} ;\left(\mathrm{A}_{2}\right) V_{A}=\left\{S \in 2^{L^{\prime} \cup C^{\prime}} \backslash\{\emptyset\} \mid \nexists d_{1} \neq\right.$ $\left.d_{2} \in S: d_{1} \sqsubseteq d_{2}\right\} ;\left(\mathrm{A}_{3}\right) v_{i}=c_{0} ;\left(\mathrm{A}_{4.1}\right)\left(n_{1}, n_{2}\right) \in \Rightarrow$ with $n_{1} \in V_{A}, n_{2} \in V_{O}$ if and only if $n_{2} \in n_{1} ;\left(\mathrm{A}_{4.2}\right)$ for any $n_{1} \in V_{O}, n_{2} \in V_{A}:\left(n_{1}, n_{2}\right) \in \Rightarrow$ if and only if $(i)$ successor covering: $\operatorname{Post}\left(\gamma\left(n_{1}\right)\right) \subseteq \gamma\left(n_{2}\right),($ ii $)$ preciseness: $\nexists n \in V_{A}$ : $\operatorname{Post}\left(\gamma\left(n_{1}\right)\right) \subseteq \gamma(n) \subset \gamma\left(n_{2}\right)$.

The following lemma states that the And-Or graph can be constructed for any WSTS and adequate domain of limits that are effective.

Lemma 1. Given a WSTS $S=\left\langle C, c_{0}, \rightarrow, \leq\right\rangle$ and an adequate domain of limits $\langle L, \sqsubseteq, \gamma\rangle$ for $\langle C, \leq\rangle$ that are effective, a finite subset $C^{\prime} \subseteq C$ with $c_{0} \in C^{\prime}$, and a finite subset $L^{\prime} \subseteq L$ with $\top \in L^{\prime}, \operatorname{Abs}\left(S, C^{\prime}, L^{\prime}\right)$ is effectively constructible.

Notice that in $\operatorname{Abs}\left(S, C^{\prime}, L^{\prime}\right)$ all the nodes have at least one successor. Indeed, for all $n \in V_{A}$, since $n \neq \emptyset$ (following point $\mathrm{A}_{4.1}$ and point $\mathrm{A}_{2}$ of Definition 6), $n$ has at least one successor. Since And-nodes are subsets of limits that may contain the $T$ element, with $\gamma(T)=C$ (following point $L_{2}$ of Definition 1), we can always approximate for any $n \in V_{O}$ the (non-empty) set of successors of $\gamma(n)$, hence we are guaranteed to have at least one successor of $n$ (point $\mathrm{A}_{4.2}$ of Definition 6).

Given a WSTS $S=\left\langle C, c_{0}, \rightarrow, \leq\right\rangle$, an associated And-Or graph $\operatorname{Abs}\left(S, L^{\prime}, C^{\prime}\right)=$ $\left\langle V_{A}, V_{O}, v_{i}, \Rightarrow\right\rangle$, and an $\leq$-upward-closed set of states $U \subseteq C$, we note $\operatorname{Abs}(U)$ the set of nodes $v \in V_{A} \cup V_{O}$ such that $\gamma(v) \cap U \neq \emptyset$, that is, the set of nodes whose associated downward-closed set of states intersects with $U$. It is easy to show that this subset of nodes can be effectively computed for any effective WSTS with adequate domain of limits.

Degenerated case If an And-Or graph is such that any Or-node has exactly one successor, the And-Or graph is said to be degenerated. In that case, the avoidability problem is equivalent to the (un)reachability problem in a plain 
graph. From the definition of $\operatorname{Abs}\left(S, C^{\prime}, L^{\prime}\right)$, we remark that the And-Or graph will be degenerated if for any $d \in C^{\prime} \cup L^{\prime}$, there exists a unique minimal set $\gamma(D)$ such that $D \in V_{A}$ and $\operatorname{Succ}(\gamma(d)) \subseteq \gamma(D)$. This motivates the next definition:

Definition 7. Given a WSTS $S=\left\langle C, c_{0}, \rightarrow, \leq\right\rangle$ and an adequate domain of limits $\langle L, \sqsubseteq, \gamma\rangle$ for $\langle C, \leq\rangle$, we say that a pair $\left\langle C^{\prime}, L^{\prime}\right\rangle$, where $C^{\prime} \subseteq C$ with $c_{0} \in C$ and $L^{\prime} \subseteq \bar{L}$ with $\top^{\prime} \in L^{\prime}$, is perfect if for any $d \in C^{\prime} \cup L^{\prime}$, there exists a unique minimal set $D \subseteq C^{\prime} \cup L^{\prime}$ such that $(i) \operatorname{Post}(\gamma(d)) \subseteq \gamma(D)$ and (ii) there is no $D^{\prime} \subseteq C^{\prime} \cup L^{\prime}$ with $\operatorname{Post}(\gamma(d)) \subseteq \gamma\left(D^{\prime}\right) \subset \gamma(D)$

Lemma 2. Given a WSTS $S=\left\langle C, c_{0}, \rightarrow, \leq\right\rangle$, an adequate domain of limits $\langle L, \sqsubseteq, \gamma\rangle$ for $\langle C, \leq\rangle$, a finite subset $C^{\prime} \subseteq C$ with $c_{0} \in C^{\prime}$, and a finite subset $L^{\prime} \subseteq L$ with $\top \in L^{\prime}$ such that $\left\langle C^{\prime}, L^{\prime}\right\rangle$ is perfect, then $\operatorname{Abs}\left(S, C^{\prime}, L^{\prime}\right)$ is a degenerated And-Or graph.

\subsection{Properties of $\operatorname{Abs}\left(S, C^{\prime}, L^{\prime}\right)$}

In this section, we prove important properties of $\operatorname{Abs}\left(S, C^{\prime}, L^{\prime}\right)$. Roughly speaking, we prove now that the abstraction we have defined above is adequate for any pair $\left\langle C^{\prime}, L^{\prime}\right\rangle$ such that $c_{0} \in C^{\prime}$ and $\top \in L^{\prime}$ (Theorem 1) and complete (Theorem 2) for some pair $\left\langle C^{\prime}, L^{\prime}\right\rangle$. To establish those results, we first show that $\operatorname{Abs}\left(S, C^{\prime}, L^{\prime}\right)$ can simulate for any $\left\langle C^{\prime}, L^{\prime}\right\rangle$ such that $c_{0} \in C^{\prime}$ and $\top \in L^{\prime}$ its underlying WSTS.

Proposition 3 (Simulation). Given a WSTS $S=\left\langle C, c_{0}, \rightarrow, \leq\right\rangle$ with an adequate domain of limits $\langle L, \sqsubseteq, \gamma\rangle$ for $\langle C, \leq\rangle$, the following holds for any $C^{\prime} \subseteq C$ with $c_{0} \in C^{\prime}$ and $L^{\prime} \subseteq L$ with $\top \in L^{\prime}$ : for any path $c_{0} c_{1} \ldots c_{k}$ of $S$ and any unfolding $T=\langle N$, root, $B, \Lambda\rangle$ of $\operatorname{Abs}\left(S, C^{\prime}, L^{\prime}\right)$ there exists a path $n_{0} n_{1} \ldots n_{2 k}$ of $T$ with $n_{0}=$ root and such that $c_{i} \in \gamma\left(\Lambda\left(n_{2 i}\right)\right)$ for $0 \leq i \leq k$.

Since any unfolding of $\operatorname{Abs}\left(S, C^{\prime}, L^{\prime}\right)$ can simulate $S=\left\langle C, c_{0}, \rightarrow, \leq\right\rangle$ for any $C^{\prime}, L^{\prime}$ with $c_{0} \in C^{\prime}$ and $\top \in L^{\prime}$, for any upward-closed set $U \subseteq C$ we know that if $\operatorname{Abs}(U)$ is avoidable in $\operatorname{Abs}\left(S, C^{\prime}, L^{\prime}\right)$ then $U$ does not intersect with $\operatorname{Reach}(S)$. That is formally stated by the next theorem.

Theorem 1 (Adequacy). Given a WSTS $S=\left\langle C, c_{0}, \rightarrow, \leq\right\rangle$, an adequate domain of limits $\langle L, \sqsubseteq, \gamma\rangle$ for $\langle C, \leq\rangle$, and an upward-closed set $U \subseteq C$, the following holds for any $C^{\prime} \subseteq C$ with $c_{0} \in C^{\prime}$ and $L^{\prime} \subseteq L$ with $\top \in \bar{L}^{\prime}$ : if $\operatorname{Abs}(U)$ is avoidable in $\operatorname{Abs}\left(S, C^{\prime}, L^{\prime}\right)$, then $\operatorname{Reach}(S) \cap U=\emptyset$.

Finally, we prove the completeness of our approach. Intuitively, the next theorem puts forward that, when the pair $\left\langle C^{\prime}, L^{\prime}\right\rangle$ is precise enough, $\operatorname{Abs}\left(S, C^{\prime}, L^{\prime}\right)$ allows us to decide negative instances of the coverability problem.

Theorem 2 (Completeness). Given a WSTS $S=\left\langle C, c_{0}, \rightarrow, \leq\right\rangle$, an adequate domain of limits $\langle L, \sqsubseteq, \gamma\rangle$ for $\langle C, \leq\rangle$ and an upward closed set $U \subseteq C$, the following holds for any $C^{\prime} \subseteq C$ with $c_{0} \in C^{\prime}$ and $L^{\prime} \subseteq L$ with $\top \in L^{\prime}$ such that $\mathrm{CS}(S) \subseteq C^{\prime} \cup L^{\prime}$ : if $\operatorname{Reach}(S) \cap U=\emptyset$ then $\operatorname{Abs}(U)$ is avoidable in $\operatorname{Abs}\left(S, C^{\prime}, L^{\prime}\right)$. 


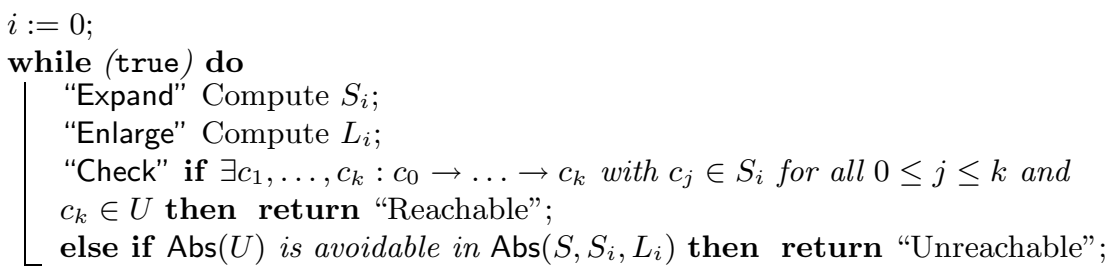

Fig. 1: Abstract algorithm Its inputs are an effective representation of a WSTS $S=\left\langle C, c_{0}, \rightarrow, \leq\right\rangle$ with the adequate limit domain $\langle L, \sqsubseteq, \gamma\rangle$ for $\langle C, \leq\rangle$ and a finite representation of the upward-closed set of states $U \subseteq C$.

\subsection{The new algorithmic schema}

Let $S_{0}, S_{1}, \ldots, S_{n} \ldots$ be an infinite sequence of finite sets of reachable states of $S$ such that $(i) \forall i \geq 0: S_{i} \subseteq S_{i+1},(i i) \forall c \in \operatorname{Reach}(S): \exists i \geq 0: c \in S_{i}$, and (iii) $c_{0} \in S_{0}$. Let $L_{0}, L_{1}, \ldots, L_{n}, \ldots$ be a infinite sequence of finite sets of limits such that $(i) \forall i \geq 0: L_{i} \subseteq L_{i+1}$, (ii) $\forall \ell \in L: \exists i \geq 0: \ell \in L_{i}$ and (iii) $\top \in L_{0}$. A schema of algorithm is given at Figure 1 and its correctness is stated in Theorem 3.

Theorem 3. For any WSTS $S$ with adequate domain of limits $\langle L, \sqsubseteq, \gamma\rangle$ that are effective, for any upward-closed set $U$ represented by $\operatorname{Min}(U)$, Algorithm at Fig. 1 terminates after a finite amount of time and returns "Reachable" if $\operatorname{Reach}(S) \cap U \neq \emptyset$, "Unreachable" otherwise.

Proof. (Sketch) If $\operatorname{Reach}(S) \cap U \neq \emptyset$, we have from Theorem 1 that $\operatorname{Abs}(U)$ is not avoidable in $\operatorname{Abs}\left(S, S_{i}, L_{i}\right)$ for all $i \geq 0$. Moreover, since for all $c \in \operatorname{Reach}(S)$ there exists $j$ such that $c \in S_{j^{\prime}}$ for all $j^{\prime} \geq j$, there exists $i \geq 0$ such that we have $c_{0} \rightarrow \ldots \rightarrow c_{k}$ with $c_{j} \in S_{i}$ for all $j$ such that $0 \leq j \leq k$ and $c_{k} \in U$. We conclude that the algorithm at Fig. 1 returns "Reachable" if $\operatorname{Reach}(S) \cap U \neq \emptyset$.

If $\operatorname{Reach}(S) \cap U=\emptyset$, we know that there exists $i \geq 0$ and a finite coverability set $\mathrm{CS}(S)$ such that $\mathrm{CS}(S) \subseteq S_{i} \cup L_{i}$. Hence, from Theorem 2 we have that $\operatorname{Abs}(U)$ is avoidable in $\operatorname{Abs}\left(S, S_{i}, L_{i}\right)$ and we conclude that the algorithm at Fig. 1 returns "Unreachable" if $\operatorname{Reach}(S) \cap U=\emptyset$.

Remark 1. Note that Theorem 3, that states the adequation and completeness of our algorithmic schema for the coverability problem of effective WSTS, is not in contradiction with the result of [6] which establishes that there does not exist a procedure that always terminates and returns a coverability set for a large class of WSTS, including ours. Indeed, to establish the correctness of our algorithm, we only need to ensure that a coverability set will be included at some point in the sequence of $S_{i}$ 's and $L_{i}$ 's. Nevertheless, given a pair $\left\langle S_{i}, L_{i}\right\rangle$, it is not possible to establish algorithmically that this pair contains a coverability set. Also, given a particular upward-closed set $U$, our algorithm may terminate before reaching a pair $\left\langle S_{i}, L_{i}\right\rangle$ that contains a coverability set, because the set $U$ is reachable or because the abstraction constructed from a pair $\left\langle S_{j}, L_{j}\right\rangle$, with $j<i$, is sufficiently precise to prove that $U$ is not reachable. 
Remark 2. Note that the constraints on the sequence of $L_{i}$ 's computed by the algorithm of Fig. 1 may be relaxed. Indeed, those constraints ensure that the algorithm eventually considers a set of limits which allows to construct a graph that is precise enough to decide negative instances of the coverability problem. However, following Theorem 2, it is sufficient to ensure that there exists $i \geq 0$ such that $S_{i} \cup L_{i}$ contains a coverability set. Hence, only the limits of a coverability set must appear in the sequence of $L_{i}$ 's.

\section{Application to Self-modifying Petri nets}

Let us show how to apply the approach proposed in the previous section to solve the coverability problem for a large subclass of Self-modifying Petri nets [14] (SMPN). SMPN are a general extension of Petri nets that includes almost all the monotonic extensions of Petri nets defined in the literature and for which, so far, there was no complete forward procedure.

\subsection{Self-modifying Petri nets}

A Self-Modifying Petri net [14], SMPN for short, is a tuple $\left\langle P, T, D^{-}, D^{+}, \mathbf{m}_{0}\right\rangle$. $P=\left\{p_{1}, \ldots, p_{k_{P}}\right\}$ is a finite (non-empty) set of places. A marking is a function $\mathbf{m}: P \rightarrow \mathbb{N}$ that assigns a natural value to each place. In the following, markings are also seen as tuples in $\mathbb{N}^{k}$ where the $i$ th dimension is the value assigned to place $p_{i} . T=\left\{t_{1}, \ldots, t_{k_{T}}\right\}$ is a finite (non-empty) set of transitions. For any $1 \leq i \leq k_{T}$ and any $1 \leq j \leq k_{P}, D_{i j}^{-}: \mathbb{N}^{k_{P}} \rightarrow \mathbb{N}$ and $D_{i j}^{+}: \mathbb{N}^{k_{P}} \rightarrow \mathbb{N}$ describe respectively the input and output effect of transition $t_{i}$ on place $p_{j}$. Namely, $D_{i j}^{-}$and $D_{i j}^{+}$are functions of the marking $\mathbf{m}$ restricted to the form $\alpha+\sum_{k=1 . . k_{P}} \beta_{k} \cdot \mathbf{m}\left(p_{k}\right)$ where $\alpha \in \mathbb{N}$ and $\beta_{k} \in \mathbb{N}$ for all $1 \leq k \leq k_{P} \cdot \mathbf{m}_{0}$ is the initial marking of the SMPN.

We define the quasi order $\preccurlyeq \subseteq \mathbb{N}^{k_{P}} \times \mathbb{N}^{k_{P}}$ on markings such that $\left\langle m_{1}, \ldots, m_{k_{P}}\right\rangle \preccurlyeq$ $\left\langle m_{1}^{\prime}, \ldots, m_{k_{P}}^{\prime}\right\rangle$ if $m_{i} \leq m_{i}^{\prime}$ for all $1 \leq i \leq k_{P}$. It is well-known that $\preccurlyeq$ is a wqo.

A transition $t_{i}$ is firable from a marking $\mathbf{m}$ if $\mathbf{m}\left(p_{j}\right) \geq D_{i j}^{-}(\mathbf{m})$ for all $p_{j} \in P$. Firing $t_{i}$ from $\mathbf{m}$ leads to a marking $\mathbf{m}^{\prime} \in \mathbb{N}^{k_{P}}$, noted $\mathbf{m} \rightarrow t_{i} \mathbf{m}^{\prime}$, such that, for any $p_{j} \in P: \mathbf{m}^{\prime}\left(p_{j}\right)=\mathbf{m}\left(p_{j}\right)+D_{i j}^{+}(\mathbf{m})-D_{i j}^{-}(\mathbf{m})$. Given a set $S$ of markings and a transition $t_{i}$, Post $\left(S, t_{i}\right)=\left\{\mathbf{m}^{\prime} \mid \exists \mathbf{m} \in S: \mathbf{m} \rightarrow t_{i} \mathbf{m}^{\prime}\right\}$.

A SMPN $\mathcal{P}$ defines a transition system $\mathcal{T}_{\mathcal{P}}=\left\langle\mathbb{N}^{k_{P}}, \mathbf{m}_{0}, \rightarrow\right\rangle$ where $\rightarrow \subseteq \mathbb{N}^{k_{P}} \times$ $\mathbb{N}^{k_{P}}$ is a transition relation and is such that we have $\left\langle\mathbf{m}, \mathbf{m}^{\prime}\right\rangle \in \rightarrow$, noted $\mathbf{m} \rightarrow \mathbf{m}^{\prime}$, if and only if there exists $t_{i} \in T$ such that $t_{i}$ is firable from $\mathbf{m}$ and $\mathbf{m} \rightarrow t_{i} \mathbf{m}^{\prime}$.

A SMPN $\mathcal{P}$ is $\preccurlyeq$-monotonic when the underlying transition system $\mathcal{T}_{\mathcal{P}}$ satisfies the monotonicity property for $\preccurlyeq$. A SMPN $\mathcal{P}$ is strongly monotonic when for every transition $t_{i}$ and markings $\mathbf{m}_{1}, \mathbf{m}_{2}$ and $\mathbf{m}_{3}$, the following holds: if $\mathbf{m}_{1} \rightarrow_{t_{i}} \mathbf{m}_{3}$ and $\mathbf{m}_{1} \preccurlyeq \mathbf{m}_{2}$, there exists $\mathbf{m}_{4}$ such that $\mathbf{m}_{2} \rightarrow t_{i} \mathbf{m}_{4}$ and $\mathbf{m}_{3} \preccurlyeq \mathbf{m}_{4}$. Obviously, all the strongly monotonic SMPN are $\preccurlyeq$-monotonic.

We say that a transition $t$ is unfirable, whenever there exists no marking $\mathbf{m}$ such that $t$ is enabled in $\mathbf{m}$. In the following, we assume that the SMPN's we 
consider do not contain unfirable transitions. The following lemma defines the syntactical subclass of SMPN's that are strongly monotonic.

Lemma 3. Given a SMPN $\mathcal{P}=\left\langle P, T, D^{-}, D^{+}, \mathbf{m}_{0}\right\rangle$ without unfirable transitions, $\mathcal{P}$ is strongly monotonic if and only if for all $t_{i} \in T, p_{j} \in P: D_{i j}^{-}=\alpha$ with $\alpha \in \mathbb{N}$ or $D_{i j}^{-}=\mathbf{m}\left(p_{j}\right)$.

Although strongly monotonic SMPN is a sub-class of SMPN, it remains a general class of monotonic systems. Indeed, almost all the monotonic extensions of Petri nets studied in the literature are syntactical sub-classes of strongly monotonic SMPN, i.e. sub-classes defined by imposing constraints on the linear expressions defining the effect of transitions. Examples of such extensions are Petri nets with transfers [5], with reset [3] and Post self-modifying Petri nets [14]. On the other hand, the other monotonic extensions of Petri nets are not syntactical sub-classes of strongly monotonic SMPN, but we can construct (in polynomial time) a strongly monotonic SMPN with the same set of places that is equivalent to the original net with respect to the coverability problem. Examples of such extensions are Petri nets with non-blocking arcs [13] and Lossy Petri nets [4]. So the algorithm that we propose in the next section is a forward algorithm that decides the coverability problem for all monotonic extensions of Petri nets proposed in the literature.

In the following, we define the adequate domain of limits we consider, state its effectiveness and show how to construct the sequences of $S_{i}$ 's and $L_{i}$ 's. Finally, we show that we always obtain degenerated And-Or graph.

\subsection{A forward algorithm to decide the coverability problem for strongly monotonic SMPN}

Domain of Limits We will consider the domain of limits $\langle\mathcal{L}, \preccurlyeq e, \gamma()$.$\rangle where \mathcal{L}=$ $(\mathbb{N} \cup\{+\infty\})^{k} \backslash \mathbb{N}^{k}, \preccurlyeq_{e} \subseteq(\mathbb{N} \cup\{+\infty\})^{k} \times(\mathbb{N} \cup\{+\infty\})^{k}$ is such that $\left\langle m_{1}, \ldots, m_{k}\right\rangle \preccurlyeq_{e}$ $\left\langle m_{1}^{\prime}, \ldots, m_{k}^{\prime}\right\rangle$ if and only if $\forall 1 \leq i \leq k: m_{i} \leq m_{i}^{\prime}$ where $c<+\infty$ for all $c \in \mathbb{N}$ ( $\leq$ is the natural order over $\mathbb{N} \cup\{+\infty\}$ ). $\gamma($.$) is defined as: \gamma(\mathbf{m})=\left\{\mathbf{m}^{\prime} \in \mathbb{N}^{k} \mid\right.$ $\left.\mathbf{m}^{\prime} \preccurlyeq_{e} \mathbf{m}\right\}$. In the following, tuples in $\mathcal{L}$ are called extended markings. It is well-known, see for instance [15], that the following lemma holds.

Lemma 4. $\langle\mathcal{L}, \preccurlyeq e, \gamma()$.$\rangle is an adequate domain of limits for \left\langle\mathbb{N}^{k}, \preccurlyeq\right\rangle$.

Notice that in this case the $T$ element such that $\gamma(\top)=\mathbb{N}^{k}$ is the marking that assigns $+\infty$ to all the places.

Given a strongly monotonic SMPN $\mathcal{P}$, we extend the underlying transition relation from markings to extended markings by assuming that $+\infty++\infty=$ $+\infty,+\infty \cdot c=+\infty$ for all $c \in \mathbb{N} \backslash\{0\}, 0 \cdot+\infty=0,+\infty+c=+\infty$ for all $c \in \mathbb{Z}$.

Since our algorithm requires the WSTS and its associated domain of limits to be effective (Definition 3), we state the following lemma :

Lemma 5. Any strongly monotonic SMPN $\mathcal{P}$ with the adequate domain of limits $\langle\mathcal{L}, \preccurlyeq e, \gamma()$.$\rangle are effective.$ 


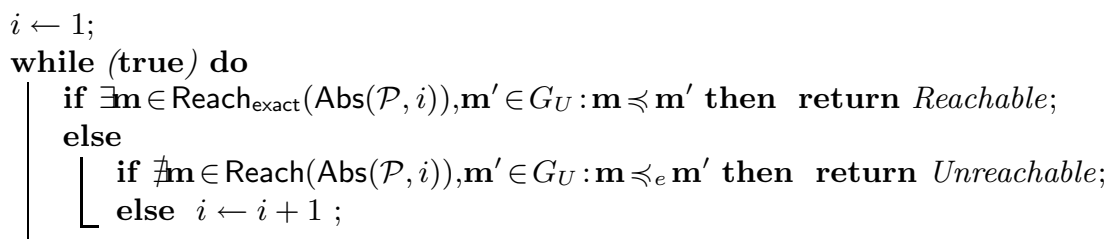

Fig. 2: A forward algorithm for SMPN Its inputs are $\mathcal{P}$, a strongly monotonic SMPN and $G_{U}$, the set of minimal elements of the $\preccurlyeq$-upward closed set $U$.

The following definition explains how we construct the $S_{i}$ 's and $L_{i}$ 's. Following Definition 6, this is sufficient to define the And-Or graphs built by our verification algorithm.

Definition 8. The sequences of $S_{i}$ 's and $L_{i}$ 's are defined as follows: $\left(\mathrm{D}_{1}\right) S_{i}=$ $\{0, \ldots, i\}^{k} \cup\left\{\mathbf{m}_{0}\right\}$, i.e. $S_{i}$ is the set of markings where each place is bounded by $i$ (plus the initial marking); $\left(\mathrm{D}_{2}\right) L_{i}=\left\{\mathbf{m} \in\{0, \ldots i,+\infty\}^{k} \mid \mathbf{m} \notin \mathbb{N}^{k}\right\}$.

It is easy to see that the $S_{i}$ 's and $L_{i}$ 's are finite sets and $(i)$ for all $i \geq 0$ : $S_{i} \subset S_{i+1}$ and $L_{i} \subset L_{i+1}$, (ii) for any $\mathbf{m} \in \mathbb{N}^{k}$, there exists $i \in \mathbb{N}$ such that for all $j \geq i: \mathbf{m} \in S_{j}$, (iii) for any $\mathbf{m} \in \mathcal{L}$, there exists $i \in \mathbb{N}$ such that for all $j \geq i: \mathbf{m} \in L_{j}$, and $(i v) \mathbf{m}_{0} \in S_{0}$ and $\top \in L_{0}$.

Degenerated And-Or graph Let us show that in the present case, one obtains a degenerated And-Or graph. For this purpose, we prove, following Lemma 2, that the pairs $\left\langle S_{i}, L_{i}\right\rangle$ are perfect pairs.

Lemma 6. Given a SMPN $\mathcal{P}=\left\langle P, T, D^{-}, D^{+}\right\rangle$with the adequate domain of limits $\left\langle\mathcal{L}, \preccurlyeq_{e}, \gamma().\right\rangle$ any pair $\left\langle S_{i}, L_{i}\right\rangle$, with $S_{i} \subseteq \mathbb{N}^{k_{P}}$ and $L_{i} \subseteq \mathcal{L}$ constructed following Definition 8, is a perfect pair.

Corollary 1. Given a strongly monotonic SMPN net $\mathcal{P}$ with the adequate domain of limits $\langle\mathcal{L}, \preccurlyeq e, \gamma()$.$\rangle and the sets S_{i} \subseteq \mathbb{N}^{k_{P}}$ and $L_{i} \subseteq \mathcal{L}$ constructed following Definition 8, $\operatorname{Abs}\left(\mathcal{P}, S_{i}, L_{i}\right)$ is a degenerated And-Or graph.

Algorithm for the coverability problem Let $\operatorname{Abs}(\mathcal{P}, i)$ be the graph (degenerated And-Or graph) $\operatorname{Abs}\left(\mathcal{P}, S_{i}, L_{i}\right)$ constructed from $\mathcal{P}, S_{i}$ and $L_{i}$. We note $\Rightarrow$ its transition relation. We define $\operatorname{Reach}_{\text {exact }}(\operatorname{Abs}(\mathcal{P}, i))$ as the set $\left\{\mathbf{m} \mid \mathbf{m}_{0} \Rightarrow \mathbf{m}_{1} \Rightarrow\right.$ $\ldots \Rightarrow \mathbf{m}_{n}$ with $\left.\forall 1 \leq j \leq n: \mathbf{m}_{j} \in S_{i}, \mathbf{m}_{n}=\mathbf{m}\right\}$ and $\operatorname{Reach}(\operatorname{Abs}(\mathcal{P}, i))$ as the set $\left\{\mathbf{m} \mid \mathbf{m}_{0} \Rightarrow \mathbf{m}_{1} \Rightarrow \ldots \Rightarrow \mathbf{m}_{n}\right.$ with $\left.\forall 1 \leq j \leq n: \mathbf{m}_{j} \in S_{i} \cup L_{i}, \mathbf{m}_{n}=\mathbf{m}\right\}$. By applying the schema presented in Section 3 to strongly monotonic selfmodifying Petri nets, we obtain the algorithm at Fig. 2. Remark that this algorithm is incremental: one can compute $\operatorname{Reach}_{\text {exact }}(\operatorname{Abs}(\mathcal{P}, i+1))$ by extending $\operatorname{Reach}_{\text {exact }}(\operatorname{Abs}(\mathcal{P}, i))$ for all $i \geq 0$. Similarly, one can construct $\operatorname{Reach}(\operatorname{Abs}(\mathcal{P}, i))$ from $\operatorname{Reach}_{\text {exact }}(\operatorname{Abs}(\mathcal{P}, i))$.

Theorem 4. For any strongly monotonic SMPN, the algorithm of Fig. 2 returns "Reachable" if $\operatorname{Reach}(\mathcal{C}) \cap U \neq \emptyset$, "Unreachable" otherwise. 


\section{Application to Lossy Channel Systems}

To show the generality of our new approach, we apply our schema of algorithm to lossy channel systems, which are systems made up of automata extended with FIFO channels that may lose messages. We recall the model, define an adequate domain of limits and show how to construct the sets $S_{i}$ 's and $L_{i}$ 's.

A Lossy Channel System, LCS for short, is a tuple $\mathcal{C}=\left\langle Q, q_{i}, F, \Sigma, T\right\rangle$ where $Q$ is a finite set of locations, $q_{i} \in Q$ is the initial location, $F$ is a finite set of channels, $\Sigma$ is a finite alphabet, $T \subseteq Q \times O p \times Q$ where $O p: F \mapsto \bigcup_{a \in \Sigma}\{? a, ! a\} \cup$ $\{$ nop $\}$. A state is a pair $\langle q, W\rangle$ where $q \in Q, W: F \mapsto \Sigma^{*}$. In the following, $\mathcal{S}_{\mathcal{C}}$ will denote the $3 \mathrm{~B}$ set of states of the LCS $\mathcal{C}$. We define the order $\precsim$ on states in $\mathcal{S}_{\mathcal{C}}$ such that for any $s=\langle q, W\rangle, s^{\prime}=\left\langle q^{\prime}, W^{\prime}\right\rangle: s \precsim s^{\prime}$ if and only if $q=q^{\prime}$ and $W(c)$ is a (not necessarily contiguous) subword of $W^{\prime}(c)$ for all $c \in F$, i.e $W(c)$ is obtained from $W^{\prime}(c)$ by deleting characters. It is well-known that $\precsim$ is a well-quasi order (see for instance [1]). A LCS $\left\langle Q, q_{i}, F, \Sigma, T\right\rangle$ defines a transition system $\left\langle\mathcal{S}_{\mathcal{C}}, s_{0}, \rightarrow\right\rangle$ where $(i) s_{0}=\left\langle q_{i}, W_{i}\right\rangle$ with $W_{i}(c)=\varepsilon$ for each $c \in F$ and (ii) $\left(\langle q, W\rangle,\left\langle q^{\prime}, W^{\prime}\right\rangle\right) \in \rightarrow$ if and only if there exists $t=\left\langle q_{1}, O p, q_{2}\right\rangle \in T$ and $\left\langle q, W^{\prime \prime}\right\rangle$ with $W^{\prime \prime} \precsim W$ such that $q=q_{1}, q^{\prime}=q_{2}$ and for all $c \in F: O p(c)=? a$ implies $W^{\prime \prime}(c)=a \cdot W^{\prime}(c)$. Furthermore, $W^{\prime}(c)=W^{\prime \prime}(c) \cdot a$ if $O p(c)=! a$ and $W^{\prime}(c)=W^{\prime \prime}(c)$ if $O p(c)=$ nop. In the following, we always consider a LCS $\mathcal{C}=\left\langle Q, q_{i}, F, \Sigma, T\right\rangle$.

Domain of limits Let $L(\Sigma)$ be the set of downward closed regular expressions (dc-re) $\left\{\left(a_{1}+\ldots+a_{n}\right)^{*} \mid \forall 1 \leq i \leq n: a_{i} \in \Sigma, \forall a_{i}, a_{j}: i \neq j\right.$ implies that $a_{i} \neq$ $\left.a_{j}\right\} \cup\{(a+\varepsilon) \mid a \in \Sigma\} \cup\{\varepsilon\}$. A simple regular expression (sre) is either a dc-re or an expression $a_{1} \cdot \ldots \cdot a_{n}$ where $\forall 1 \leq i \leq n: a_{i}$ is a dc-re. The size of a sre is the number of dc-re that compose it. The set of limits is $\mathcal{L}(\Sigma, Q)=$ $\left\{\langle q, E\rangle \mid q \in Q, E: F \mapsto L(\Sigma)^{*}\right.$ assigns a sre to each channel $\left.{ }^{2}\right\} \cup\{\top\}$. For $\langle q, E\rangle \in \mathcal{L}(\Sigma, Q) \backslash\{\varepsilon\}: \llbracket\langle q, E\rangle \rrbracket$ denotes the set of pairs $\langle q, W\rangle \in \mathcal{S}_{\mathcal{C}}$ such that $W(c)$ is a word in the language generated by the regular expression $E(c)$ for all $c \in F$. We define the function $\gamma: \mathcal{S}_{\mathcal{C}} \cup \mathcal{L}(\Sigma, Q) \rightarrow 2^{\mathcal{S}_{\mathcal{C}}}$ such that $(i)$ for all $\langle q, W\rangle \in \mathcal{S}_{\mathcal{C}}: \gamma(\langle q, W\rangle)=\left\{\left\langle q, W^{\prime}\right\rangle \mid\left\langle q, W^{\prime}\right\rangle \precsim\langle q, W\rangle\right\},(i i) \gamma(\top)=\{\langle q, W\rangle \mid$ $q \in Q, W(c) \in \Sigma^{*}$ for all $\left.c \in F\right\}$ and $($ iii $)$ for all $\langle q, E\rangle \in \mathcal{L}(\Sigma, Q) \backslash\{\top\}$ : $\gamma(\langle q, E\rangle)=\llbracket\langle q, E\rangle \rrbracket$. We define $\sqsubseteq:\left(\mathcal{S}_{\mathcal{C}} \cup \mathcal{L}(\Sigma, Q)\right) \times\left(\mathcal{S}_{\mathcal{C}} \cup \mathcal{L}(\Sigma, Q)\right)$ as follows : $c_{1} \sqsubseteq c_{2}$ if and only if $\gamma\left(c_{1}\right) \subseteq \gamma\left(c_{2}\right)$.

It is easy to see that $(\mathcal{L}(\Sigma, Q), \sqsubseteq, \gamma)$ is an adequate domain of limits for $\left(\mathcal{S}_{\mathcal{C}}, \precsim\right)$ and that any LCS $\mathcal{C}$ with this domain of limits is effective.

Construction of the $S_{i}$ 's and the $L_{i}$ 's We construct the sequences of the $S_{i}$ 's and $L_{i}$ 's as follows. $S_{i}=\left\{\langle q, W\rangle \in \mathcal{S}_{\mathcal{C}} \mid q \in Q, \forall c \in F: W(c)=\varepsilon\right.$ or $W(c)=$ $a_{1} \cdot \ldots \cdot a_{n}$ with $n \leq i$ and $\left.\forall 1 \leq j \leq n: a_{j} \in \Sigma\right\}$, i.e. $S_{i}$ is the set of states where the contents of the channels are words of size at most $i$. Similarly, $L_{i}=$ $\left\{\langle q, E\rangle \in \mathcal{L}(\Sigma, Q) \mid \forall c \in F: E(c)=\varepsilon\right.$ or $E(c)=e_{1} \cdot \ldots \cdot e_{n}$ with $n \leq i$ and $\forall 1 \leq$

\footnotetext{
${ }^{2}$ We also require that $E$ does not assign $\varepsilon$ to all the channels because we require in Definition 1 that the set of limits be disjoint from $\mathcal{S}_{\mathcal{C}}$.
} 
$\left.j \leq n: e_{j} \in L(\Sigma)\right\} \cup\{\top\}$, i.e. $L_{i}$ is the set of limits that assign sre of size at most $i$ to the channels (plus the $T$ element).

It is not difficult to see that the sequences of $S_{i}$ 's and $L_{i}$ 's satisfy the hypothesis of the algorithm of Fig. 1.

\section{Conclusion}

In this paper, we have defined a new approach to solve the coverability problem of WSTS, which we call "Expand, Enlarge and Check". When applied to a large class of monotonic counter systems (the strong monotonic Self-modifying Petri nets), our approach produces an algorithm that uses forward analysis to decide the coverability problem. Up to now, such a forward approach was known only for Petri nets (the Karp and Miller algorithm), a restricted subclass of strong monotonic SMPN. We have demonstrated the generality of our approach by showing how to apply the algorithmic schema to lossy channel systems.

\section{References}

1. P. A. Abdulla, K. Cerans, B. Jonsson, and Y.-K. Tsay. General Decidability Theorems for Infinite-state Systems. In Proc. LICS'96, pages 313-321. IEEE, 1996.

2. P.A. Abdulla and B. Jonsson. Verifying Programs with Unreliable Channels. In Proc. LICS'93, pages 160-170. IEEE, 1993.

3. T. Araki and T. Kasami. Some decision problems related to the reachability problem for petri nets. Theoretical Computer Science, 3(1):85-104, 1977.

4. A. Bouajjani and R. Mayr. Model Checking Lossy Vector Addition Systems. In Proc. STACS'99, LNCS 1563, pages 323-333. Springer, 1999.

5. G. Ciardo. Petri nets with marking-dependent arc multiplicity: properties and analysis. In Proc. ICATPN 94, LNCS 815, pages 179-198. Springer, 1994.

6. C. Dufourd, A. Finkel, and Ph. Schnoebelen. Reset Nets Between Decidability and Undecidability. In In Proc. ICALP'98, LNCS 1443, pages 103-115. Springer, 1998.

7. J. Esparza, A. Finkel, and R. Mayr. On the Verification of Broadcast Protocols. In Proc. LICS'99, pages 352-359. IEEE, 1999.

8. E. A. Emerson and K. S. Namjoshi. On Model Checking for Non-deterministic Infinite-state Systems. In Proc. LICS '98, pages 70-80. IEEE, 1998.

9. A. Finkel, J.-F. Raskin, M. Samuelides, and L. Van Begin. Monotonic Extensisions of Petri Nets : Forward and Backward Search Revisited. In Proc. INFINITY'02, ENTCS 68(6). Elsevier, 2002.

10. A. Finkel and P. Schnoebelen. Well-structured transition systems everywhere! Theoretical Computer Science, 256(1-2):63-92, 2001.

11. S.M. German and A.P. Sistla. Reasoning about systems with many processes. JACM 39(3): 675-735, 1992.

12. T. A. Henzinger, O. Kupferman, and S. Qadeer. From prehistoric to postmodern symbolic model checking. Formal Methods in System Design, 23(3):303-327, 2003.

13. J.-F. Raskin and L. Van Begin. Petri Nets with Non-blocking Arcs are Difficult to Analyse. In Proc. INFINITY'03, ENTCS 96. Elsevier, 2003.

14. R. Valk. On the computational power of extended petri nets. In Proc. MFCS'78, LNCS 64, pages 527-535. Springer, 1978.

15. L. Van Begin. Efficient Verification of Counting Abstractions for Parametric systems. PhD thesis, Université Libre de Bruxelles, Belgium, 2003. 